\title{
Efficacy and safety of ultrasound-guided above-knee lateral approach for popliteal sciatic nerve block in surgeries below the knee: a randomized controlled trial
}

\author{
Lin-Jia Zhü, Chan-Juan Gong", Zhen-Feng Zhang, Qing-Wei Zhang, Pei-Pei Peng, Yan Ni \\ Department of Anesthesiology and Perioperative Medicine, The First Affiliated Hospital with Nanjing Medical University, Nanjing, China \\ Contributions: (I) Conception and design: Y Ni; (II) Administrative support: QW Zhang, PP Peng; (III) Provision of study materials or patients: LJ \\ Zhu, CJ Gong; (IV) Collection and assembly of data: ZF Zhang; (V) Data analysis and interpretation: LJ Zhu, ZF Zhang; (VI) Manuscript writing: \\ All authors; (VII) Final approval of manuscript: All authors. \\ "These authors contributed equally to this work. \\ Correspondence to: Yan Ni, MD. Department of Anesthesiology and Perioperative Medicine, The First Affiliated Hospital of Nanjing Medical \\ University, 300 Guangzhou Road, Nanjing 210029, China. Email: niyan812@njmu.edu.cn.
}

\begin{abstract}
Background: Ultrasound guidance has become a standard method for detection of nerve structures in regional anesthesia. During ultrasound-guided blockade, to identify anatomical structures is crucial but can be challenging. In clinical practice, we find a wide difference in the visibility score of the sciatic nerve (SN) through different approaches. This study aimed to compare SNB through the anterior and above-knee lateral approach in terms of identification ease, performance efficacy, and safety.

Methods: Patients scheduled for below-knee surgery were randomized to either receive SNB using the above-knee lateral approach (Group L, n=27) or the anterior approach (Group A, n=26). The primary outcome was the visibility score of SN. Secondary outcomes included the time taken to identify the SN, nerve depth, success rate of SN identification, number of needle passes, time to elicit foot flexion, needle depth, and occurrence of SNB complications. Additionally, the sensory block onset and analgesia duration were assessed.
\end{abstract}

Results: We included 53 adult patients. Compared with Group A, Group L showed a higher SN visibility score $[3.25(3.17,3.67)$ vs. $2.50(1.86,2.68), \mathrm{P}<0.001]$. The scan time was significantly shorter in Group L [8.70 (6.01) s vs. $31.54(11.87) \mathrm{s}, \mathrm{P}<0.001]$. The depth of the $\mathrm{SN}$ was $3.20(0.56) \mathrm{cm}$ in Group L and $5.53(0.84) \mathrm{cm}$ in Group A $(\mathrm{P}<0.001)$, and the needle insertion depth was $7.15(0.90) \mathrm{cm}$ in group L and $8.32(1.13) \mathrm{cm}$ in Group A $(\mathrm{P}<0.001)$. The number of needle passes was less in Group L, as well as the time to elicit foot flexion, and the time taken to perform the $\mathrm{SN}$ block (all $\mathrm{P}<0.001$ ). The success rate of $\mathrm{SN}$ identification was non-significantly higher in Group L. There was no significant between-group difference in the onset of sensory block, as well as postoperative analgesia duration. None of the approaches involved acute systemic toxicity and hematoma occurrence.

Conclusions: Based on the visibility score, the above-knee lateral approach allowed easy SN identification and safe SNB. Using the ultrasound-guided above-knee lateral approach for SNB in below-knee surgeries could be a reliable choice.

Keywords: Sciatic nerve; ultrasound-guided; above-knee lateral approach; anterior approach; below-knee surgeries

Submitted Jan 03, 2021. Accepted for publication Mar 28, 2021.

doi: $10.21037 /$ apm-21-10

View this article at: http://dx.doi.org/10.21037/apm-21-10 


\section{Introduction}

For below-knee surgeries, combining popliteal sciatic nerve block (SNB) and adductor canal block (ACB) can effectively provide anesthesia and postoperative analgesia $(1,2)$. Ultrasound-guided techniques are reliable for SNB. Although ultrasound guidance is helpful for sciatic nerve (SN) localization, the identification of SN from other anatomical structures, can be challenging, but is a basic requirement for an effective and safe ultrasound-guided nerve block. Owing to a relatively deep location in the proximal lower limb and anisotropy of the $\mathrm{SN}$, the visibility score of $\mathrm{SN}$ was the lowest in peripheral nerves (3), which may complicate the nerve anatomical distinction from the surrounding tissues (4). Therefore, there are differences in the ease of performance, reliability, and safety across different approaches.

Posterior popliteal SNB, which is the most frequently used approach, requires extra effort and exacerbates pain, especially in patients who have difficulty in repositioning. Previous studies have described a reliable lateral approach to the popliteal nerve block, which is employed either with the patient positioned in "gapped supine" or flexing the knee with the transducer placed under the fossa (5). However, there may be initial confusion regarding how to move the needle to approach the nerve, which impedes transducer placement and manipulation. The anterior approach for $\mathrm{SNB}$ is often applied to patients who prefer lying supine. In this approach, the SN was situated beneath the adductor magnus muscle, lateral to the long head of the biceps femoris muscle, and posteromedial to the lesser trochanter. The anatomical features of the anterior block hinder the success of SN identification, render it time-consuming and technically demanding as an advanced nerve block $(6,7)$. In our clinical practice, the transducer is perpendicularly placed on the skin at approximately $10 \mathrm{~cm}$ proximal to the lateral femoral condyle with the patient positioned supine. Further, the operated leg is extended at the knee joint with the foot positioned perpendicularly to the bed. This is known as the above-knee lateral approach, which has been previously used through anatomical landmark identification or under a nerve stimulator. In this approach, the $\mathrm{SN}$ was just located beneath the vastus lateralis and the biceps femoris. In addition, with less influence on the strength of hamstrings, and anatomical feature with spherical shape, this approach has recently regained interest with ultrasound application. However, owing to the difference in the visibility score of the SN through different approaches, it remains unclear which approach is easier and more accurate in identifying the SN and performing SNB. This study aimed to compare the efficacy and safety between the above-knee lateral approach and anterior approach in patients undergoing below-knee surgeries. We present the following article in accordance with the CONSORT reporting checklist (available at: http://dx.doi.org/10.21037/ apm-21-10).

\section{Methods}

This prospective randomized control trial was conducted in the First Affiliated Hospital with Nanjing Medical University, Nanjing, China. After getting approval from the Ethics Committee of our hospital (No. 2019-SR-398) and registering in the Chinese clinical trial (trial registration: ChiCTR2000029486), we recruited seventy patients scheduled for below-knee surgeries between March 2, 2020 and August 30, 2020. Before enrollment, all patients or their legal representatives provided written informed consent. Our study conformed to the provisions of the Declaration of Helsinki (as revised in 2013) (available at https://www. wma.net/wp-content/uploads/2016/11/DoH-Oct2013JAMA.pdf).

\section{Setting and subjects}

The inclusion criteria were as follows: (I) age 18-64 years; (II) American Society of Anesthesiology Physical Status I or II; and (III) body mass index $18-26 \mathrm{~kg} / \mathrm{m}^{2}$; (IV) anticipated duration of operation less than $90 \mathrm{~min}$. We excluded patients with a history of allergy to local anesthetics, contraindications to regional anesthesia, diabetic neuropathy, preoperative coagulation dysfunction, chronic analgesic treatment, or damage of the sciatic and branch nerves.

We randomly assigned the patients to receive a sciatic nerve blockade either using the above-knee lateral approach (Group L) or anterior approach (Group A) with ultrasound guidance. Group allocation was conducted using a random permuted block method. All the patients underwent presurgery fasting without premedication. All the patients underwent pre-surgery fasting without premedication. Upon arrival at the pre-anesthesia room, the patients underwent routine standard monitoring, including electrocardiography, non-invasive blood pressure, and pulse oximetry using a Mindray T6 monitor. Before conducting the nerve block, intravenous access was established followed by intravenous administration of midazolam (1 mg) and fentanyl $(0.05 \mathrm{mg})$ for anxiolysis and analgesia. This was followed by oxygen 

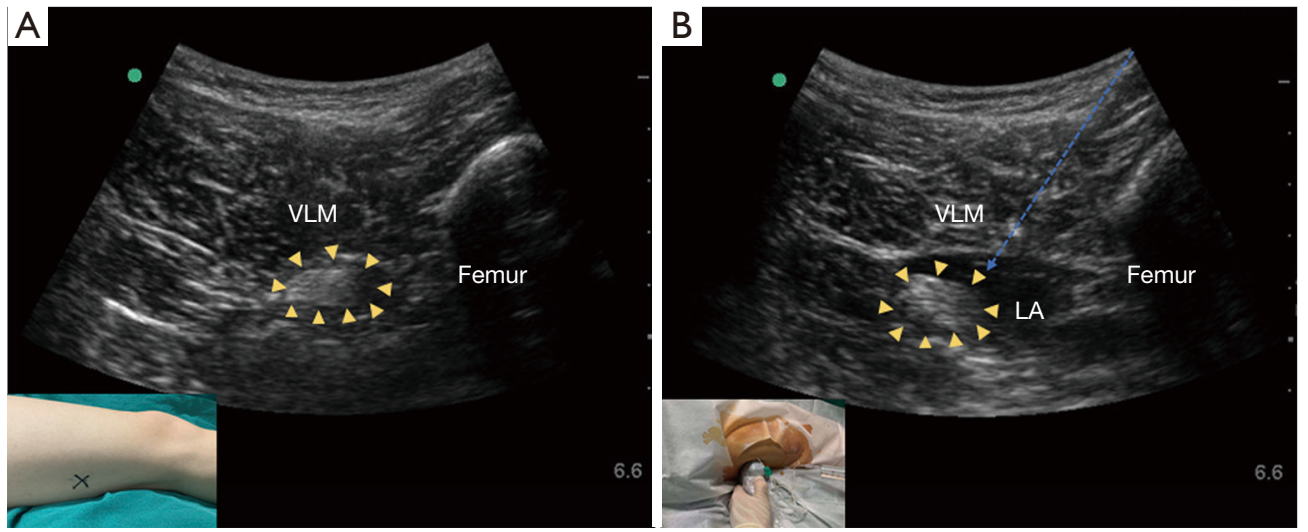

Figure 1 Ultrasound images obtained during SNB with an ultrasound-guided above-knee lateral approach. (A) A pre-procedure ultrasound image obtained with the transducer positioned perpendicular to the skin approximately $10 \mathrm{~cm}$ proximal to the lateral femoral condyle. The $\mathrm{SN}$ is seen as a hyperechoic thick oval structure (yellow arrow heads); (B) An ultrasound image obtained immediately after injection of local anesthetics in proximity to the SN (yellow arrow heads). The needle is indicated by a blue dotted line. LA, local anesthetics; VLM, vastus lateralis muscle; $\mathrm{SN}$, sciatic nerve; $\mathrm{SNB}$, sciatic nerve block.

administration at a $3 \mathrm{~L} / \mathrm{min}$ rate via a face mask. A SonoSite ultrasound system (FUJIFILM SonoSite Edge I Ultrasound System) with a low-frequency curved-array transducer (rC60xi/5-2 MHz) and a high-frequency linear transducer (HFL38xi/13-6 MHz) were used for SNB and ACB, respectively. We used a nerve stimulator (Stimuplex HNS 11, B. BRAUN AG) to confirm SN location and guarantee SNB safety. An experienced attending physician in our regional anesthesia team performed all the blocks using an in-plane technique. After all the blocks were completed, opaque adhesive plasters were placed on the needle insertion points of both approaches. A blinded investigator assessed the sensory and motor blockade.

\section{Ultrasound-guided above-knee lateral approach}

For the above-knee lateral approach to the SN, the patient was placed in the supine position with the operated leg extended at the knee joint and the foot positioned perpendicular to the bed. Above-knee SN visualization was performed using a low-frequency curved-array transducer. After skin sterilization, the ultrasound transducer was positioned perpendicular to the skin approximately $10 \mathrm{~cm}$ proximal to the lateral femoral condyle (5) using a sterile ultrasound gel and plastic transducer cover. Upon femur identification through ultrasound, the transducer position was carefully manipulated to obtain the clearest $\mathrm{SN}$ view, which was posterior to the femur, as well as between the vastus lateralis and biceps femoris (Figure 1A). An ultrasound image of the SN was then obtained and stored. Subsequently, the injection site was infiltrated with $2 \mathrm{~mL}$ of $1 \%$ lidocaine. A short-bevel, 100-mm 22-gauge needle (Stimuplex D; B. BRAUN AG), which was attached to a nerve stimulator, was advanced toward the $\mathrm{SN}$ under ultrasound guidance. The nerve stimulator was set at a pulse duration of $0.1 \mathrm{msec}$ using an initial stimulating current and frequency of $1.0 \mathrm{~mA}$ and $2 \mathrm{~Hz}$, respectively. Once the needle tip was placed close to the $\mathrm{SN}$ with knee dorsiflexion or plantar flexion being elicited, the needle tip position was further adjusted to confirm the loss of knee dorsiflexion or plantar flexion at a current of $0.4 \mathrm{~mA}$. After negative aspiration, we injected $20 \mathrm{~mL}$ of $0.375 \%$ ropivacaine around the $\mathrm{SN}$ (Figure 1B).

\section{Ultrasound-guided anterior approach}

The patient was placed in the supine position; further, the hip on the operated side was slightly flexed and externally rotated. After skin sterilization, the transducer was placed perpendicularly to the skin on the medial mid-thigh using a sterile ultrasound gel and transducer cover (8). Upon identification of the femur, the transducer was slightly adjusted to allow clear SN visualization, which was located at the medial side of the femur beneath the adductor magnus muscle (Figure 2A). An ultrasound image of the $\mathrm{SN}$ was saved. After asepsis confirmation and skin infiltration with lidocaine $1 \%$, a 100-mm 22-gauge or 120-mm 22-gauge (if needed) connected to the nerve stimulator was inserted toward the 

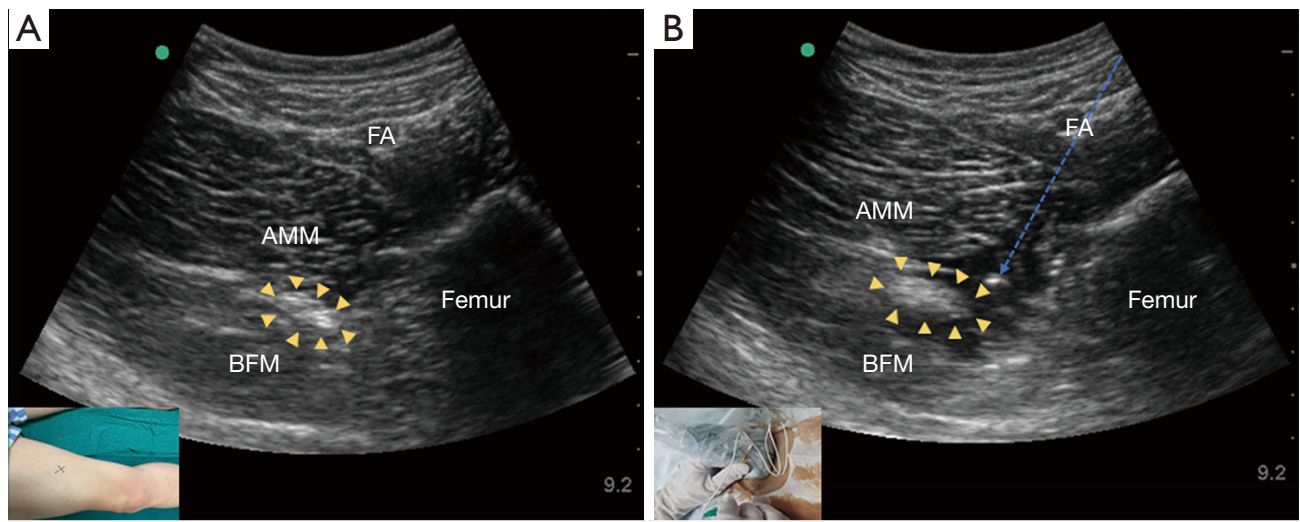

Figure 2 Ultrasound images obtained during SNB with an ultrasound-guided anterior approach. (A) A pre-procedure ultrasound image obtained with the transducer placed perpendicularly to the skin on the medial mid-thigh, with the hip slightly flexed and externally rotated. The SN is seen as a hyperechoic thick oval structure (yellow arrow heads) (B) An ultrasound image obtained immediately after injection of local anesthetics in proximity to the SN (yellow arrow heads). The needle is indicated by a blue dotted line. AMM, adductor magnus muscle; BFM, biceps femoris muscle; FA, femoral artery; LA, local anesthetics; SN, sciatic nerve; SNB, sciatic nerve block.

$\mathrm{SN}$ in a lateral-to-medial direction with ultrasound guidance. The nerve stimulator settings were similar to those for the lateral approach. After negative aspiration, $20 \mathrm{~mL}$ of $0.375 \%$ ropivacaine was injected (Figure 2B).

\section{Outcomes}

The SN visibility score was evaluated by four assessors who were experienced in US-guided regional anesthesia to eliminate visual and subjective biases. All the assessors were blinded to the approach. The visibility scores were determined using a 6-point visibility scale: 0 , no nerve identified; 1 , nerve identified with high probability; 2 , nerve identified but most of it not visible; 3 , nerve identified and $>50 \%$ of its borders precisely distinguished from surrounding structures; 4 , nerve completely visible but fascicles poorly defined; and 5 , nerve completely visible and multiple fascicles identifiable (9).

During the SNB procedure, a nurse anesthetist recorded the scan time (from transducer placement to $\mathrm{SN}$ identification), SN depth (distance from the skin to the superficial SN surface), time taken to elicit foot plantarflexion or dorsiflexion, time taken to perform the SN block (from needle insertion to withdrawal), needle depth (distance from the skin to the needle tip), number of needle passes (deliberate needle tip withdrawal to skin level or additional skin puncture), the visual analogue scale (VAS), and occurrence of SNB complications such as inadvertent vessel puncture and local anesthetic systemic toxicity. The success of $\mathrm{SN}$ identification was confirmed by successfully eliciting knee dorsiflexion or plantar flexion. Next, an ACB was performed using $10 \mathrm{~mL}$ of ropivacaine $0.375 \%$ under real-time ultrasound guidance.

A blinded investigator assessed the sensory and motor blockade on the operated lower leg at 5-min intervals for $45 \mathrm{~min}$. Using a pinprick with a blunted needle, sensory blockade was assessed for the tibial nerve (foot sole) and common peroneus nerve (foot dorsal area). Motor function was examined by assessing the patients' ability to perform knee plantar or dorsal flexion (0, no movement; 1 , light movement; 2, normal movement) (10). We recorded the time required for the onset of sensory and motor blockades (from local anesthetic administration to the start of sensory and motor changes, respectively). A successful block was defined as complete sensory block of the CPN and TN within $45 \mathrm{~min}$. In case of block failure, general anesthesia was administered for the surgery. After SNB assessment, the patients were transferred to the operation room where they received intravenous dexmedetomidine $(1 \mu \mathrm{g} / \mathrm{kg}$ pumped within $10 \mathrm{~min}$, followed by $0.1 \mu \mathrm{g} / \mathrm{kg} / \mathrm{h}$. Propofol-based anesthetics were administered in case of complaints of an unbearable tourniquet reaction. Postoperatively, our acute pain service group recorded the sensory block duration (from local anesthetic administration to full sensory recovery).

\section{Methylene blue staining of detached limb}

With the patients' consent, methylene blue staining of SN 
using the above-knee lateral approach was implemented in the detached limb of two patients scheduled for hip disarticulation. A 100-mm 22-gauge needle was used for the injection. After the needle tip was confirmed under ultrasound guidance to be in close proximity to the $\mathrm{SN}$, $20 \mathrm{~mL}$ of $0.1 \%$ methylene blue (Jichuan Pharmaceutical Co. Ltd.) was injected. Approximately 10 minutes after injection, the detached limb was placed in a prone position, and the posterior aspect of the popliteal fossa was dissected to assess the spread of the injectate.

\section{Sample size calculation}

Based on the pilot study with 10 patients in each group, we obtained the mean between-group difference in the visibility score of the $\mathrm{SN}$ (the mean difference between two groups: 0.7 ). Two-tailed statistical analysis required 55 patients in two groups with a type-I error risk of 0.05 and power of 0.8 . To account for a dropout rate of $20 \%$, we enrolled 70 patients. Group allocation was performed using an online randomization program and concealed in opaque envelopes by the study coordinator prior to patient enrollment. The anesthesiologist performing the SNB opened the envelope just before the patient entered the pre-anesthesia room. The study patients, intraoperative anesthesia, surgical teams, and the study assessors were all blinded to the allocation.

\section{Statistical analysis}

Statistical analyses were performed using statistical software SPSS (version 22.0; SPSS Inc., IBM Corporation, Armonk, NY, USA) and GraphPad Prism version 7 (GraphPad Software, Inc., San Diego, CA, USA). Data were expressed as mean \pm standard deviation or median with $95 \%$ confidence interval (CI) after checking the normality with the Shapiro-Wilk test. Between-group variance analysis was performed using the two-sample Student's $t$-test for continuous parametric variables, including time to perform SNB, duration of analgesia and demographics profiles. For categorical variables, including the success rate of SN identification and complete blockade of SN, we used Pearson's chi-squared or Fisher's exact tests. Time to elicit flexion, and onset of sensory and motor block, were analyzed using the two-sample Mann-Whitney $U$ rank-sum test. Between-group comparisons of stratified variables, like number of needle pass, were performed using the rank-sum test. Statistical significance was set at two-sided $\mathrm{P}<0.05$.

\section{Results}

\section{Basic patient data and characteristics}

Seventy patients scheduled for below-knee surgeries were recruited. Among them, 53 patients (27 in Group L and 26 in Group A) completed the study. Among the 17 patients (8 in Group L and 9 in Group A), 11 patients were previously excluded due to uncontrolled diabetes, pre-existing neurological damage, and preference for general anesthesia; the remaining 6 patients ( 3 in each group) were eliminated for data loss due to time constraints of the block assessment (Figure 3). There were no between-group differences in patient demographics, surgery type, and surgery duration (Table 1).

\section{Comparison of SNB-related parameters between the two groups}

Compared with Group A, Group L showed a higher visibility score $[3.25(3.17,3.67)$ vs. $2.50(1.86,2.68)$, $\mathrm{P}<0.001]$ (Figure 4). The scan time required for $\mathrm{SN}$ identification was significantly shorter in Group L ( $8.70 \pm 6.01$ s vs. $31.54 \pm 11.87$ s, $\mathrm{P}<0.001)$ (Figure 5). The $\mathrm{SN}$ depth was $3.20(0.56)$ and $5.53(0.84)$ in Group L and A, respectively $(\mathrm{P}<0.001)$; further, the needle insertion depth was $7.15(0.90)$ and $8.32(1.13) \mathrm{cm}$ in Group L and Group A, respectively $(\mathrm{P}<0.001)$ (Figure 6). Compared with Group L, Group A showed a fewer number of needle passes [number of needle pass (0/1/2/3/: 22/5/0/0 vs. 8/10/7/1)], less time to elicit foot plantarflexion or dorsiflexion [35.00 (32.19, $46.32) \mathrm{s} v s .64 .56(62.28,104.33) \mathrm{s}]$, and less time taken to perform the SN block $(49.70 \pm 5.97 v s .71 .50 \pm 11.66 \mathrm{~s}$ ) (all $\mathrm{P}<0.001)$. The success rate of $\mathrm{SN}$ identification was nonsignificantly higher in Group L (88.9\% vs. 69.2\%, $\mathrm{P}=0.078)$. During the puncture, there was no significant betweengroup difference in the visual analog scale (VAS) score. Four patients in Group A presented inadvertent vessel puncture; however, none of the patients showed systemic toxicity from local anesthetics. There was no between-group difference in the onset of sensory block for peroneal [11.00 (10.05, $13.65) \mathrm{s} v s .10 .00(10.98,14.79) \mathrm{s}, \mathrm{P}=0.683]$ and tibial nerves [15.00 (13.18,17.56) s vs. $15.00(13.23,18.30) \mathrm{s}, \mathrm{P}=0.963]$, as well as the onset of motor block for peroneal [20.00 (17.88, $22.12)$ s vs. $22.50(20.15,24.46) \mathrm{s}, \mathrm{P}=0.126]$ and tibial nerves [20.00 (19.16, 23.80) s vs. $25.00(21.47,24.30) \mathrm{s}, \mathrm{P}=0.240]$. One patient in Group L and two patients in Group A were 


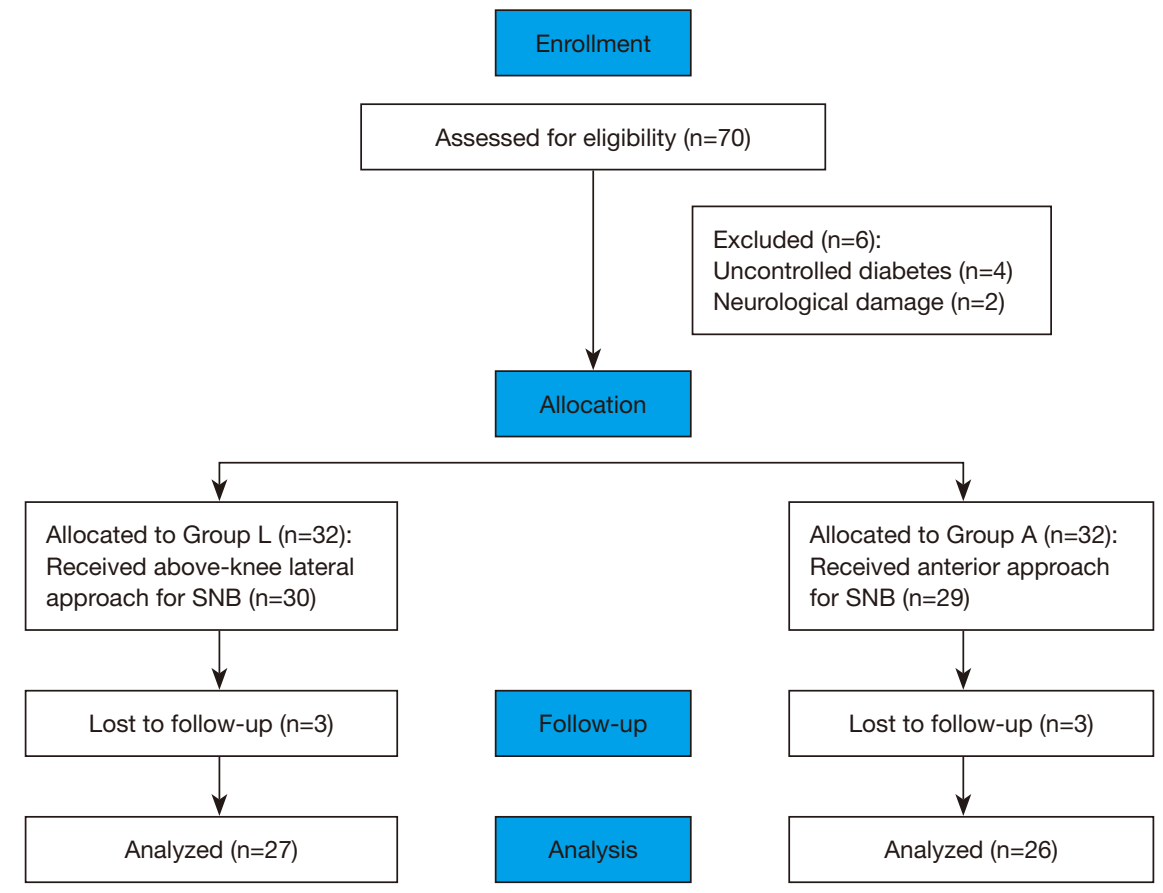

Figure 3 Flowchart diagram of the study. SNB, sciatic nerve block.

Table 1 Demographics parameters of participants in the two groups

\begin{tabular}{lccc}
\hline Variables & Group L $(\mathrm{n}=27)$ & Group A $(\mathrm{n}=26)$ & $\mathrm{P}$ \\
\hline Age $(\mathrm{y})$ & $42.67 \pm 13.38$ & $43.54 \pm 15.12$ & 0.825 \\
Gender $(\mathrm{M} / \mathrm{F})$ & $19 / 8$ & $16 / 10$ & 0.497 \\
ASA class (I/II/III) & $20 / 7 / 0$ & $17 / 8 / 1$ & 0.449 \\
BMI $\left(\mathrm{kg} / \mathrm{m}^{2}\right)$ & $23.76 \pm 3.01$ & $24.17 \pm 3.44$ & 0.648 \\
Types of surgery, $\mathrm{n}(\%)$ & & & 0.496 \\
Surgical debridement & $4(14.82)$ & $3(11.54)$ & $13(50.00)$ \\
Fracture of the ankle & $15(55.56)$ & $10(38.46)$ & $60[57,69]$ \\
Hallux valgus & $8(29.63)$ & $60[58,71]$ & 0.993 \\
Duration of operation (min) &
\end{tabular}

ASA, American Society of Anesthesiologists; BMI, body mass index.

switched to general anesthesia. There was no betweengroup difference in the frequency of complete sensory blockade of the SN at 45 minutes $(96.30 \%$ vs. $92.30 \%$, $\mathrm{P}=0.973)$ and analgesia duration $(20.31 \pm 5.19$ vs. $20.34 \pm 3.66$, $\mathrm{P}=0.089$ ) (Table 2).

\section{Evaluation of the detached limb}

We confirmed that the popliteal SN could be stained sufficiently by methylene blue using the above-knee lateral approach in the 2 detached limbs (Figure 7).

\section{Discussion}

The SN at the popliteal location blocks the anterior, lateral, and posterior lower leg, ankle, and foot (2). Combining popliteal SNB with ACB has been widely used to provide anesthesia and analgesia for below-knee surgeries, especially 


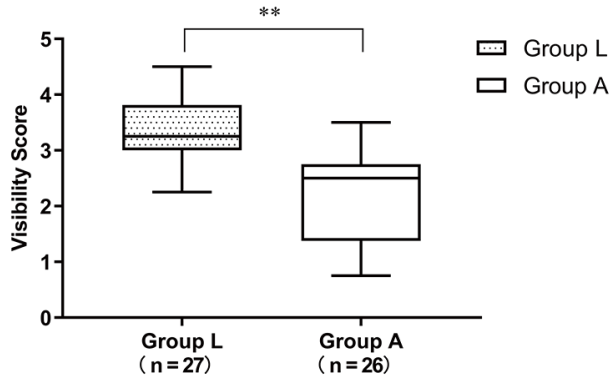

Figure 4 Visibility score of the $\mathrm{SN}$ in either the lateral above-knee approach or the anterior approach. ${ }^{* *} \mathrm{P}<0.001$. SN, sciatic nerve.

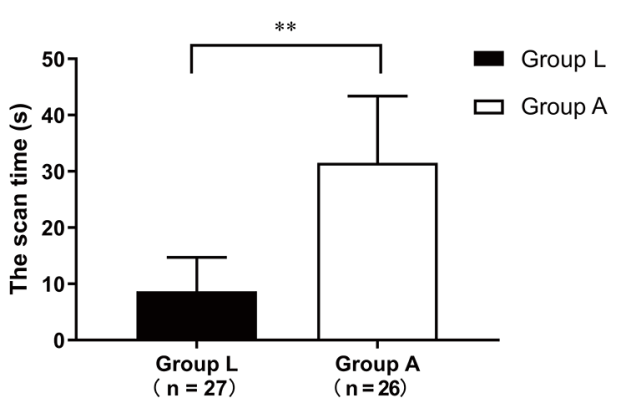

Figure 5 The scan time used to identify the $\mathrm{SN}$ in either the lateral above-knee approach or the anterior approach. ${ }^{* *} \mathrm{P}<0.001$. $\mathrm{SN}$, sciatic nerve.
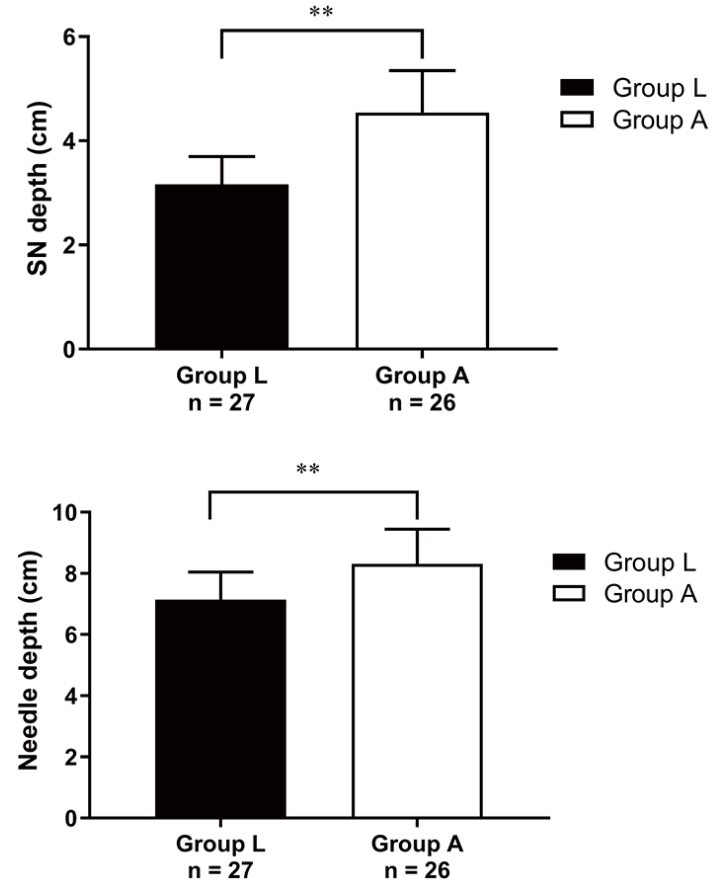

Figure 6 The SN depth and needle depth in either the lateral above-knee approach or the anterior approach. ${ }^{* *} \mathrm{P}<0.001$. SN, sciatic nerve.

Table 2 SNB-related parameters and adverse events

\begin{tabular}{|c|c|c|c|}
\hline Variables & Group L ( $n=27)$ & Group A $(n=26)$ & $\mathrm{P}$ \\
\hline Time to elicit flexion (s) & 35 [32.19-46.32] & 64.56 [62.28-104.33] & $<0.001$ \\
\hline Time to perform SNB (s) & $49.70 \pm 5.97$ & $71.50 \pm 11.66$ & $<0.001$ \\
\hline Success rate of SN identification, n (\%) & $24(88.9)$ & $18(69.2)$ & 0.078 \\
\hline Inadvertent vessel puncture & $0(0)$ & $4(15.4)$ & 0.110 \\
\hline Systemic toxicity & $0(0)$ & $0(0)$ & 1.000 \\
\hline \multicolumn{4}{|l|}{ Onset of sensory block } \\
\hline Peroneal nerve & $11[10.05,13.65]$ & $10[10.98,14.79]$ & 0.683 \\
\hline Peroneal nerve & $20[17.88,22.12]$ & $22.5[20.15,24.46]$ & 0.126 \\
\hline Tibial nerve & $20[19.16,23.80]$ & $25[21.47,24.30]$ & 0.240 \\
\hline Complete blockade of SN, n (\%) & $26(96.30)$ & $24(92.30)$ & 0.973 \\
\hline Duration of analgesia $(\mathrm{h})$ & $20.31 \pm 5.19$ & $20.34 \pm 3.66$ & 0.089 \\
\hline
\end{tabular}

SN, sciatic nerve; SNB, sciatic nerve block. 


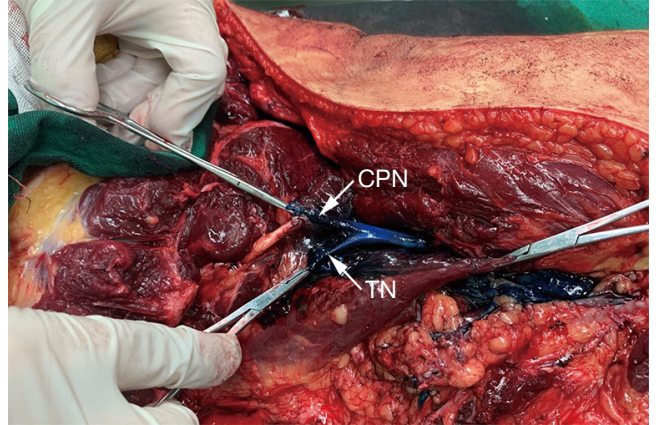

Figure 7 Spread of methylene blue in a detached limb using the ultrasound-guided above-knee lateral approach for SNB. Both the $\mathrm{CFN}$ and $\mathrm{TN}$ are surrounded by methylene blue. CFN, common peroneal nerve; $\mathrm{TN}$, tibial nerve.

foot and ankle surgeries (11). SNB can be performed using an ultrasound machine or a nerve stimulator (12). Since a 2-dimensional conventional ultrasound machine may have limited ability to distinguish the SN from the surrounding tissue (13), a convenient and easy approach for increasing the reliability of nerve localization is required. This study applied ultrasound-guided SNB in below-knee surgeries using either the above-knee lateral or anterior approach. Based on the sonographic visibility score, scan time, nerve depth, time to perform SNB, success rate of SN identification, number of needle passes, and block onset and duration, we found that the ultrasound-guided above-knee lateral approach could facilitate SNB without affecting the blockade onset and quality.

Several approaches for SNB have been previously described with the classic posterior approach in the popliteal fossa being the most common. However, the posterior approach for ultrasound-guided popliteal SNB is only used when the patient is in the lateral or prone position, which might limit its use in some patients. The anterior approach, which was introduced in 1963, allows SNB in the supine position (14). However, this approach is considered an advanced technique since the SN depth and anisotropy may impede visualization (6,7). Kim et al. compared several different positions for $\mathrm{SN}$ identification and reported that the anterior approach had an approximate visibility score of 2.5 (3), which was consistent with our results. Given the deep SN location from the anterior approach, the $\mathrm{SN}$ can be easily confounded by surrounding muscles and falsely identified through ultrasound imaging. Barrington et al. reported that $37.5 \%$ of the patients had a poor or average image quality (15), which is similar to our results. In this study, 10 of the 26 patients who underwent the anterior approach obtained a poor image of the $\mathrm{SN}$ (visibility score $<2$ ).

The popliteal lateral approach to the $\mathrm{SN}$ has been reported as feasible without requiring lifting of the leg by extending the hip for block performance (5). Numerous studies have shown that the lateral approach to the popliteal fossa, which is an effective alternative as for the posterior popliteal approach, could allow SNB performance with the patients in the supine position. However, this approach often requires more than one stitch; further, there is a risk of vascular puncture when only employing guidance using a nerve stimulator. The lateral popliteal SNB can be performed with the patient positioned in the "gapped supine" and "elevated leg" position with the ultrasound transducer placed under the popliteal fossa (5). However, this might increase the technical difficulty and require a longer performance time. In this study, the above-knee lateral approach involved positioning of the transducer perpendicular to the lateral skin at approximately $10 \mathrm{~cm}$ proximal to the lateral femoral condyle. This approach allowed a better visibility score of the $\mathrm{SN}$; moreover, the $\mathrm{SN}$ was more superficial, which resulted in easier and quicker ultrasound imaging.

As aforementioned, the anterior approach for SNB is considered time-consuming and technically demanding $(6,7)$. In this approach, the puncture route often involves the common and deep femoral arteries or veins. Caution is required to avoid inadvertent vascular damage. Therefore, in the anterior approach, even when the SN image is clear, the operator should still redirect the needle to avoid vascular puncture. Contrastingly, there was a low number of needle passes adjusted for SNB in the aboveknee lateral approach. During the puncture procedure, no patient presented local anesthetic-related systemic toxicity; however, four patients in Group A, but none in Group L, suffered from inadvertent vessel puncture. Moreover, there was no between-group difference in the success rate of $\mathrm{SNB}$, as well as SB onset and duration. These findings could be attributed to the technique of combining ultrasound and nerve stimulator, which might improve the SNB success rate and safety by avoiding vascular structures and allowing direct observation of local anesthetic spread (16). Although there were between-group differences in the number of needle pass and procedure duration, there was no betweengroup difference in the VAS during the puncture. This might be associated with the prophylactic use of midazolam and fentanyl for sedation and analgesia.

This study has several limitations. First, we only compared the anterior and above-knee lateral approaches 
with patients positioned in the supine position. Second, under ultrasound guidance, a local anesthetic was injected perineurally rather than within the para-neural sheath. Previous studies have reported that SNB using the classical popliteal posterior approach could cause faster onset, longer duration, and higher success rate $(17,18)$. However, we did not compare the aforementioned approaches with the classical popliteal posterior approach. This alternative approach allows injection of local anesthetics within the para-neural sheath to achieve a circumferential spread around the tibial and peroneal nerves under ultrasound guidance, which might allow a quicker and more successful block $(19,20)$. Third, we excluded patients with BMI $>26 \mathrm{~kg} / \mathrm{m}^{2}$; moreover, there is a need to determine whether the above-knee lateral approach is more suitable for these patients.

\section{Conclusions}

In conclusion, under ultrasound guidance, the anterior and above-knee lateral approaches can be used to perform SN block in patients undergoing below-knee surgeries, especially for those preferring the supine position. However, the above-knee lateral approach block allows for a better visibility score of the ultrasound image and quicker SNB performance. These findings are encouraging and support the use of the ultrasound-guided above-knee lateral approach for SNB in below-knee surgeries.

\section{Acknowledgments}

We would like to thank Editage (www.editage.com) for English language editing.

Funding: None.

\section{Footnote}

Reporting Checklist: The authors have completed the CONSORT reporting checklist. Available at: http://dx.doi. org/10.21037/apm-21-10

Data Sharing Statement: Available at: http://dx.doi. org/10.21037/apm-21-10

Conflicts of Interest: All authors have completed the ICMJE uniform disclosure form (available at: http://dx.doi. org/10.21037/apm-21-10). The authors have no conflicts of interest to declare.
Ethical Statement: The authors are accountable for all aspects of the work in ensuring that questions related to the accuracy or integrity of any part of the work are appropriately investigated and resolved. This study was approved by the Institutional Ethics Committee of the $1^{\text {st }}$ Affiliated Hospital of Nanjing Medical University, Nanjing, China (No. 2019-SR-398) and was registered in the Chinese clinical trial (NO.: ChiCTR2000029486). Before enrollment, all patients or their legal representatives provided written informed consent. Our study conformed to the provisions of the Declaration of Helsinki (as revised in 2013), available at https://www.wma.net/wp-content/ uploads/2016/11/DoH-Oct2013-JAMA.pdf.

Open Access Statement: This is an Open Access article distributed in accordance with the Creative Commons Attribution-NonCommercial-NoDerivs 4.0 International License (CC BY-NC-ND 4.0), which permits the noncommercial replication and distribution of the article with the strict proviso that no changes or edits are made and the original work is properly cited (including links to both the formal publication through the relevant DOI and the license). See: https://creativecommons.org/licenses/by-nc-nd/4.0/.

\section{References}

1. Fournier R, Weber A, Gamulin Z. Posterior labat vs. lateral popliteal sciatic block: posterior sciatic block has quicker onset and shorter duration of anaesthesia. Acta Anaesthesiol Scand 2005;49:683-6.

2. Wiederhold BD, Garmon EH, Peterson E, et al. Nerve Block Anesthesia. StatPearls. Treasure Island (FL): StatPearls Publishing Copyright (C 2020, StatPearls Publishing LLC.; 2020.

3. Kim HJ, Chin KJ, Kim H, et al. Ultrasound-Guided Anterior Approach to a Sciatic Nerve Block: Influence of Lower Limb Positioning on the Visibility and Depth of the Sciatic Nerve. J Ultrasound Med 2020;39:1641-7.

4. Birnbaum J, Diederich L, Ertmer M, et al. A new score for characterizing the visibility of anatomical structures during ultrasound guided regional anesthesia: a retrospective cohort study. Minerva Anestesiol 2020;86:922-9.

5. Creech C, Meyr AJ. Techniques of popliteal nerve regional anesthesia. J Foot Ankle Surg 2013;52:681-5.

6. Yoshida T, Nakamoto T, Hashimoto C, et al. An Ultrasound-Guided Lateral Approach for Proximal Sciatic Nerve Block: A Randomized Comparison With the Anterior Approach and a Cadaveric Evaluation. Reg 
Anesth Pain Med 2018;43:712-9.

7. Yektaş A, Balkan B. Comparison of sciatic nerve block quality achieved using the anterior and posterior approaches: a randomised trial. BMC Anesthesiol 2019;19:225.

8. Kendir S, Torun B, Akkaya T, et al. Re-defining the anatomical structures for blocking the nerves in adductor canal and sciatic nerve through the same injection site: an anatomical study. Surg Radiol Anat 2018;40:1267-74.

9. Frkovic V, Ward C, Preckel B, et al. Influence of arm position on ultrasound visibility of the axillary brachial plexus. Eur J Anaesthesiol 2015;32:771-80.

10. Triadó VD, Crespo MT, Aguilar JL, et al. A comparison of lateral popliteal versus lateral midfemoral sciatic nerve blockade using ropivacaine $0.5 \%$. Reg Anesth Pain Med 2004;29:23-7.

11. Gianakos AL, Romanelli F, Rao N, et al. Combination Lower Extremity Nerve Blocks and Their Effect on Postoperative Pain and Opioid Consumption: A Systematic Review. J Foot Ankle Surg 2021;60:121-31.

12. Eldegwy MH, Ibrahim SM, Hanora S, et al. Ultrasoundguided sciatic politeal nerve block: a comparison of separate tibial and common peroneal nerve injections versus injecting proximal to the bifurcation. Middle East J Anaesthesiol 2015;23:171-6.

13. Choquet O, Capdevila X. Case report: Three-dimensional high-resolution ultrasound-guided nerve blocks: a new

Cite this article as: Zhu LJ, Gong CJ, Zhang ZF, Zhang QW, Peng PP, Ni Y. Efficacy and safety of ultrasound-guided above-knee lateral approach for popliteal sciatic nerve block in surgeries below the knee: a randomized controlled trial. Ann Palliat Med 2021;10(5):5188-5197. doi: 10.21037/apm-21-10 panoramic vision of local anesthetic spread and perineural catheter tip location. Anesth Analg 2013;116:1176-81.

14. Beck GP. Anterior approach to sciatic nerve block. Anesthesiology 1963;24:222-4.

15. Barrington MJ, Lai SL, Briggs CA, et al. Ultrasoundguided midthigh sciatic nerve block-a clinical and anatomical study. Reg Anesth Pain Med 2008;33:369-76.

16. Gray AT, Collins AB, Schafhalter-Zoppoth I. Sciatic nerve block in a child: a sonographic approach. Anesth Analg 2003;97:1300-2.

17. Bang SU, Kim DJ, Bae JH, et al. Minimum effective local anesthetic volume for surgical anesthesia by subparaneural, ultrasound-guided popliteal sciatic nerve block: A prospective dose-finding study. Medicine (Baltimore) 2016;95:e4652.

18. Cappelleri G, Ambrosoli AL, Gemma M, et al. Intraneural Ultrasound-guided Sciatic Nerve Block: Minimum Effective Volume and Electrophysiologic Effects. Anesthesiology 2018;129:241-8.

19. Yamamoto H, Sakura S, Wada M, et al. A prospective, randomized comparison between single- and multipleinjection techniques for ultrasound-guided subgluteal sciatic nerve block. Anesth Analg 2014;119:1442-8.

20. Gürkan Y, Ohtaroğlu ÇN. Can we use "coin sign" image to predict block success after performance of sciatic nerve block? Agri 2012;24:142-3. 